\title{
LINEAR FUNCTIONAL EQUATIONS WITH ALGEBRAIC PARAMETERS
}

\author{
GERGELY KISS
}

\begin{abstract}
We describe the solutions of linear functional equations with algebraic parameters.
\end{abstract}

\section{INTRODUCTION}

Let $K$ be a subfield of the field of complex numbers. We investigate the functional equation

$$
\sum_{i=1}^{n} a_{i} f\left(b_{i} x+y\right)=0
$$

where $a_{i} \in \mathbb{C}, b_{i} \in K$ are given and $f: \mathbb{C} \rightarrow \mathbb{C}$ is the unknown function. We shall also consider the more general equation

$$
\sum_{i=1}^{n} a_{i} f\left(b_{i} x+c_{i} y\right)=0
$$

where $a_{i} \in \mathbb{C}, b_{i}, c_{i} \in K$ are given and $f: \mathbb{C} \rightarrow \mathbb{C}$ is the unknown function. Our aim is to describe the solutions, at least for some classes of these equations, on the field $K$. Note that every solution defined on $K$ can be extended to a solution on $\mathbb{C}$. Indeed, since $\mathbb{C}$ is a linear space over the field $K$, the identity on $K$ can be extended to $\mathbb{C}$ as a linear function over $K$. Let $\phi: \mathbb{C} \rightarrow K$ be such an extension. It is clear that if $f$ satisfies (2) for every $x, y \in K$, then $f \circ \phi$ satisfies (2) for every $x, y \in \mathbb{C}$.

In this paper we shall consider those equations of the form (2) for which the solutions are generalized polynomials on $K$.

Date: February 21, 2014.

1991 Mathematics Subject Classification. Primary 39B99, 11R04.

Key words and phrases. Linear functional equations, field isomorphisms, variety. Partially supported by Hungarian Scientific Foundation grant no. 72655. 
By a generalized polynomial on an Abelian group $A$ we mean a function $f: A \rightarrow \mathbb{C}$ such that, for a suitable $n$, we have

$$
\Delta_{h_{1}} \ldots \Delta_{h_{n+1}} f(x)=0
$$

for every $h_{1}, \ldots, h_{n+1}, x \in A$. Here $\Delta_{h}$ denotes the difference operator,

$$
\Delta_{h} f(x)=f(x+h)-f(x) .
$$

The smallest $n$ for which there are elements $h_{1}, \ldots, h_{n+1}$ satisfying (3) is called the degree of $f$. We shall restrict our attention to those equations (2) whose parameters satisfy the following condition.

The numbers $a_{1}, \ldots, a_{n}$ are nonzero, and there is an $1 \leq i \leq n$ such that $b_{i} c_{j} \neq b_{j} c_{i}$ holds for any $1 \leq j \leq n, j \neq i$.

The following result is an easy consequence of [8] (see also [1], [5] and [9]):

Theorem 1.1. Suppose (4). If

$$
\sum_{i=1}^{n} a_{i} f\left(b_{i} x+c_{i} y\right)=0
$$

is true for every $x, y \in K$, then $f$ is a generalized polynomial on $K$ of degree at most $n-2$.

Note that if $c_{i}=1$ for every $i=1, \ldots, n$, then (4) is satisfied whenever $a_{1}, \ldots, a_{n} \neq 0$ and the numbers $b_{1}, \ldots, b_{n}$ are distinct. That is, equation (1) with different $b_{1}, \ldots, b_{n}$ always satisfies condition (4), and thus its solutions are generalized polynomials.

In Section 2 our aim is to describe the solutions when $b_{i}$ and $c_{i}$ are algebraic numbers. We show that in this case the space of the solutions of (2) is spanned by the injective homomorphisms (shortly isomorphisms) of $K$ or the products of them. (See Theorem 2.3, Theorem 2.7.)

These results make it possible, at least in principle, to find every solution of a functional equation satisfying condition (4) when $b_{i}, c_{i}$ are algebraic numbers, since the number of these isomorphisms and of their product is finite.

We say that the equation (2) is trivial if every additive function is a solution of the functional equation. Examples of trivial equations are

$$
3 f(x+\sqrt{2 y})-f(6 x+(3 \sqrt{2}+5 \sqrt{3}) y)+5 f(x+\sqrt{3} y)=0
$$

and

$$
2 f(x+y)-f(x+2 y)-2 f(x+\pi y)+f(x+2 \pi y)=0 .
$$


In Section 3 we prove that the trivial equations also have the property that the set of their additive solutions is spanned by those solutions which are injective homomorphisms. This amounts to show that the set of all additive functions is spanned by injective homomorphisms (3.1).

In Section 4 we show that, in general, the set of additive solutions is not always spanned by the injective homomorphism solutions. In Theorem 4.3 we prove that there are equations with this property for every field $K$ containing transcendental numbers, showing that Theorem 2.3 is best possible.

Note that the set of the solutions of (2) is a linear space over $\mathbb{C}$, but not necessary translation invariant with respect to the addition. There are important cases such as (1) with distinct $b_{1}, \ldots, b_{n}$, when the space of solutions is translation invariant. (In general, if the points $\left(b_{i}, c_{i}\right) \in \mathbb{C}^{2}$ lie on a line not going through the origin $(0,0)$, then the space of solutions is translation invariant.) Translation invariance sometimes simplifies the description of the space of solutions (see, e.g. Remark 2.9).

We remark that the results presented here were motivated by the paper [4].

\section{TheOREMS IN ALGEBRAIC CASE}

The proofs of this Section are based on some results of polynomialexponential functions on groups.

Let $(G, *)$ be an Abelian group, and let $\mathbb{C}^{G}$ denote the linear space of all complex valued functions defined on $\mathrm{G}$ equipped with the product topology. By a variety on $G$ we mean a translation invariant closed linear subspace of $\mathbb{C}^{G}$. We say that the function $f: G \rightarrow \mathbb{C}$ is additive, if $f$ is a homomorphism of $G$ into the additive group of $\mathbb{C}$. A function is a polynomial if it belongs to the algebra generated by the constant functions and the additive functions. A nonzero function $m \in \mathbb{C}^{G}$ is called an exponential if $m$ is multiplicative; that is, if $m(x * y)=$ $m(x) \cdot m(y)$ for every $x, y \in G$. An exponential monomial is the product of a polynomial and an exponential, a polynomial-exponential function is a finite sum of exponential monomials.

Lemma 2.1. Let $(G, *)$ be an Abelian group, $V$ be a translation invariant linear subspace of $C(G)$, and let $\sum_{i=1}^{M} p_{i} \cdot m_{i} \in V$, where $p_{1}, \ldots, p_{M}$ are nonzero generalized polynomials and $m_{1}, \ldots, m_{i}$ are distinct nonzero 
exponentials on $G$. Then $\left(\Delta_{h_{1}} \ldots \Delta_{h_{k}} p_{i}\right) \cdot m_{i} \in V$ for every $i$ and for every $h_{1}, \ldots, h_{k} \in G$.

If $p_{i}$ is not constant, then there is a nonzero additive function $A$ : $G \rightarrow \mathbb{C}$ such that $A \cdot m_{i} \in V$ and $m_{i} \in V$.

Proof. Since $m_{i}$ is a nonzero exponential, $m_{i}(x) \neq 0$ for any $x \in G$. If $p(x) m(x) \in V$, then $p(x * h) m(x * h)$ and $c \cdot p(x) m(x)$ is in $V$ for any $c \neq 0 \in \mathbb{C}$, because $V$ is a translation invariant linear space. Thus, the equation

$$
\begin{aligned}
& p(x * h) m(x * h)-m(h) p(x) m(x)= \\
= & (p(x * h)-p(x)) m(x) m(h)=\left(\Delta_{h} p(x)\right) \cdot m(x) m(h)
\end{aligned}
$$

shows that $\Delta_{h} p(x) m(x) \in V$. The iteration of this process proves the first statement of the lemma. It is well known that for every generalized polynomial $p$ there exist an integer $k$ and an additive function $A$ such that $\Delta_{h_{1}} \ldots \Delta_{h_{k}} p=A$ and $\Delta_{h_{1}} \ldots \Delta_{h_{k}} \Delta_{h_{k+1}} p$ is a nonzero constant for some $h_{1}, \ldots, h_{k}, h_{k+1} \in G$. Thus, if $p \cdot m \in V$, then $A \cdot m$ and $m$ are in $V$, which completes the proof.

The following proposition will be used frequently (see [3, Theorem 14.5.1.]).

Proposition 2.2. Let $K \subset \mathbb{C}$ be a finitely generated field and $\phi: K \rightarrow$ $\mathbb{C}$ be an injective homomorphism. Then there exists an automorphism $\psi$ of $\mathbb{C}$ such that $\left.\psi\right|_{K}=\phi$.

Theorem 2.3. Let $b_{1}, \ldots, b_{n}, c_{1}, \ldots, c_{n}$ be algebraic numbers satisfying $(4)$, and put $K=\mathbb{Q}\left(b_{1}, \ldots, b_{n}\right)$. Then every additive solution of (2) defined on $K$ is of the form

$$
d_{1} \phi_{1}+\cdots+d_{k} \phi_{k}
$$

where $d_{1}, \ldots, d_{k}$ are complex numbers and $\phi_{1}, \ldots, \phi_{k}: K \rightarrow \mathbb{C}$ are injective homomorphisms satisfying

$$
\sum_{i=1}^{n} a_{i} \phi_{j}\left(b_{i}\right)=0 \text { and } \sum_{i=1}^{n} a_{i} \phi_{j}\left(c_{i}\right)=0
$$

for every $j \in\{1, \ldots, k\}$.

Remark 2.4. It is clear that all injective homomorphisms satisfying (5) are solutions of (2).

Proof. If $f$ is an additive solution of (2) on $K$, then

$$
0=\sum_{i=1}^{n} a_{i} f\left(b_{i} x+c_{i} y\right)=\sum_{i=1}^{n} a_{i} f\left(b_{i} x\right)+\sum_{i=1}^{n} a_{i} f\left(c_{i} y\right)
$$


for every $x, y \in K$. By substituting $x=0$ and $y=0$ we get $\sum_{i=1}^{n} a_{i} f\left(c_{i} y\right)=$ 0 and $\sum_{i=1}^{n} a_{i} f\left(b_{i} x\right)=0$, respectively.

Let $V$ denote the set of additive functions $f: K \rightarrow \mathbb{C}$ satisfying

$$
\sum_{i=1}^{n} a_{i} f\left(b_{i} h\right)=0 \text { and } \sum_{i=1}^{n} a_{i} f\left(c_{i} h\right)=0
$$

for every $h \in K$. We denote $K^{*}=\{x \in K: x \neq 0\}$; then $K^{*}$ is an Abelian group under multiplication. Let

$$
V^{*}=\left\{\left.f\right|_{K^{*}}: f \in V\right\} \text {. }
$$

We prove that $V^{*}$ is a variety on the Abelian group $K^{*}$.

It is clear that $V^{*}$ is a linear space and that $V^{*}$ is translation invariant. Indeed, if $f$ is additive and satisfies (6), then $x \rightarrow f(a x)$ is also additive, and also satisfies (6) for every $a \in K^{*}$. Let $g: K^{*} \rightarrow \mathbb{C}$ be a function in the closure of $V^{*}$. Extend $g$ by $g(0)=0$. Let $a, b \in K^{\star}$ be such that $a+b$ is also in $K^{*}$, i.e. $a+b \neq 0$. Since $g \in \mathrm{cl} V^{*}$, for every $\varepsilon>0$ there exists $f \in V^{*}$ such that

$$
|f(a)-g(a)|<\varepsilon,|f(b)-g(b)|<\varepsilon \text { and }|f(a+b)-g(a+b)|<\varepsilon .
$$

Now, $f(a)+f(b)=f(a+b)$ because $f$ is additive, so $\mid g(a+b)-g(a)-$ $g(b) \mid<3 \varepsilon$. This is true for every $\varepsilon$, therefore $g(a+b)=g(a)+g(b)$. This holds for every $a, b \in K$ with $a, b, a+b \neq 0$ and then, by $g(0)=0$, it follows that $g$ is additive on $K$. A similar argument shows that $g$ satisfies (6). This means that $V^{*}$ is closed, and thus $V^{*}$ is a variety.

Since the $b_{i}^{\prime}$ s are algebraic numbers, the field $K$ is a finite algebraic extension of the field $\mathbb{Q}$. If $\beta_{1}, \ldots, \beta_{N}$ is a basis of $K$ as a linear space over $\mathbb{Q}$ and if $f: K \rightarrow \mathbb{C}$ is additive, then

$$
f\left(r_{1} \beta_{1}+\ldots+r_{N} \beta_{N}\right)=r_{1} f\left(\beta_{1}\right)+\ldots+r_{N} f\left(\beta_{N}\right)
$$

for every $r_{1}, \ldots, r_{N} \in \mathbb{Q}$. Therefore, the values of $f$ at any point of $K$ are determined by the values $f\left(\beta_{1}\right), \ldots, f\left(\beta_{N}\right)$. Since the set of all functions mapping $\left\{\beta_{1}, \ldots, \beta_{N}\right\}$ into $\mathbb{C}$ has dimension $N$ it follows that the set $\mathcal{A}$ of all additive functions defined on $K$ forms a finite dimensional vector space over $\mathbb{C}$. This implies that $V$, as a linear subspace of $\mathcal{A}$ is also finite dimensional. Consequently, $V^{*}$ is a finite dimensional translation invariant linear space of functions defined on $K^{*}$. By [8] and [2], it follows that every element of $V^{*}$ is a polynomial-exponential function.

In other words, every element $f \in V^{*}$ can be written as a finite sum $f=\sum_{j=1}^{M} p_{j} \cdot m_{j}$, where $p_{1}, \ldots, p_{M}$ are nonzero polynomials and $m_{1}, \ldots, m_{M}$ are distinct exponentials on $K^{*}$. 
By Lemma 2.1, $m_{1}, \ldots, m_{M} \in V^{*}$. Therefore,

$$
m_{j}(x y)=m_{j}(x) m_{j}(y)
$$

for every $j$ and $x, y \in K^{*}$. If we define $m_{j}(0)=0$ then $m_{j}$ becomes additive on $K$, and thus $m_{j}$ is a homomorphism of the field $K$ into $\mathbb{C}$. Since $m_{j} \not \equiv 0$, it must be an injective homomorphism. The condition $m_{j} \in V^{*}$ implies that $m_{j}$ is a solution of (2), and thus satisfies (5).

We prove that each $p_{j}$ is constant.

Suppose that $p_{1}$ is not constant. Then, by Lemma 2.1, there is a nonzero additive function $A$ on $K^{*}$ such that $A \cdot m_{1} \in V^{*}$. Due to Proposition 2.2, if $K \subset \mathbb{C}$ is a finitely generated field and $\phi: K \rightarrow \mathbb{C}$ is an injective homomorphism, then there exists an automorphism $\psi$ of $\mathbb{C}$ such that $\left.\psi\right|_{K}=\phi$. Therefore, $m_{1}$ can be extended to $\mathbb{C}$ as an automorphism. We shall denote the extension also by $m_{1}$.

We put $d=m_{1}^{-1} \circ\left(A \cdot m_{1}\right)$ and $d(0)=0$. We show that $d$ is a derivation on $K$. Since $m_{1}$ is an automorphism, it is an additive function, and then $m_{1}^{-1}$ is also additive. Since $A \cdot m_{1} \in V^{*}$, thus $A \cdot m_{1}$ also additive, and the composition $d$ is also additive; that is, $d(x+y)=d(x)+d(y)$. Since $A$ is additive on $K^{*}$ with respect to the multiplication, we have $A(x y)=A(x)+A(y)$ for every $x, y \in K^{*}$. Therefore,

$$
\begin{aligned}
d(x y) & =\left(m_{1}^{-1} \circ\left(A \cdot m_{1}\right)\right)(x y)=m_{1}^{-1}\left(A(x y) \cdot m_{1}(x y)\right)= \\
& =m_{1}^{-1}\left((A(x)+A(y)) \cdot m_{1}(x) m_{1}(y)\right)= \\
& =m_{1}^{-1}\left(A(x) \cdot m_{1}(x)\right) \cdot y+m_{1}^{-1}\left(A(y) \cdot m_{1}(y)\right) \cdot x= \\
& =d(x) y+d(y) x
\end{aligned}
$$

for every $x, y \in K^{*}$. Clearly, $d(x y)=d(x) y+d(y) x$ holds in the cases $x=0$ or $y=0$ as well. Therefore, $d$ is a derivation on $K$.

On the other hand, it is well known (and it is easy to check) that on algebraic extensions of $\mathbb{Q}$ the only derivation is the identically zero function. However, the function $A$ is not identically zero and $m_{1}$ is an automorphism, thus $d$ cannot be identically zero. This contradiction shows that $p_{1}$ must be constant, which completes the proof.

Now we are interested in the solutions of (1) of arbitrary degree. In order to generalize Theorem 2.3 we need some technical lemmas.

Let $K$ be a subfield of $\mathbb{C}$. A function $f_{k}: K \rightarrow \mathbb{C}$ is called a monomial of degree $k$, if there exists a nonzero, symmetric and $k$-additive function $F_{k}: K^{k} \rightarrow \mathbb{C}$ such that $f_{k}(x)=F_{k}(x, \ldots, x)$ for every $x \in \mathbb{C}$. It is wellknown that if $f$ is a generalized polynomial of degree $m$, then it can 
be written of the form $f=f_{0}+\sum_{k=1}^{m} f_{k}$, where $f_{0}$ is constant and $f_{k}$ is a monomial of degree $k(k=1, \ldots, m)$ (see [6]).

Lemma 2.5. Suppose that $f=f_{0}+\sum_{k=1}^{m} f_{k}$ is a solution of $(2)$, where $f_{0}$ is constant and $f_{k}$ is a monomial of degree $k(k=1, \ldots, m)$. Then each of $f_{0}, \ldots, f_{m}$ is a solution of $(2)$.

Proof. [4, Lemma 2.1.]

Lemma 2.6. Let $f(x)=F_{k}(\underbrace{x, \ldots, x}_{k})$ be a monomial, where $F_{k}$ is symmetric and $k$-additive. In this case

$$
k ! F_{k}\left(x_{1}, x_{2}, \ldots, x_{k}\right)=\Delta_{x_{1}} \Delta_{x_{2}} \ldots \Delta_{x_{k}} f(t)
$$

for every $t \in \mathbb{C}$.

Proof. See $[3, \S 9$, Lemma 2.]

Theorem 2.7. Let $b_{i}, c_{i}(i=1, \ldots, n)$ be algebraic numbers satisfying (4), and put $K=\mathbb{Q}\left(b_{1}, \ldots, b_{n}, c_{1}, \ldots, c_{n}\right)$. Let $f$ be a solution of (2) defined on $K$. If the degree of $f$ is at most $k$, then $f$ is the linear combination of products of at most $k$ injective homomorphisms of $K$ which products are also solutions of (2).

Proof. Suppose that there is a solution of (2) of degree $k$. By Lemma 2.5 , there is a solution which is a monomial of degree $k$. Let $f_{k}(x)=$ $F_{k}(x, \ldots, x)$ be a solution, where $F_{k}$ is nonzero, symmetric and $k$ additive. Our aim is to show that $F_{k}$ is the linear combination of functions of the form $\phi_{1} \cdots \phi_{k}$, where $\phi_{1}, \ldots, \phi_{k}: K \rightarrow \mathbb{C}$ are injective homomorphisms such that the terms of the linear combination are solutions of (2) as well.

Let $V$ be the set of all functions $S: K^{k} \rightarrow \mathbb{C}$ such that $S$ is $k$ additive and the function $x \mapsto S\left(s_{1} x, s_{2} x, \ldots, s_{k} x\right)(x \in K)$ satisfies (2) for every $s_{1}, s_{2}, \ldots s_{k} \in K$. We prove that $F_{k} \in V$. By Lemma 2.6,

$$
k ! \cdot F_{k}\left(s_{1} x, s_{2} x, \ldots, s_{k} x\right)=\Delta_{s_{1} x} \Delta_{s_{2} x} \ldots \Delta_{s_{k} x} f_{k}(0)=\sum_{j=1}^{M} \pm f_{k}\left(e_{j} x\right)
$$

with suitable $e_{1}, \ldots, e_{M} \in K$. Thus $F_{k}\left(s_{1} x, s_{2} x, \ldots, s_{k} x\right)$ is a linear combination of functions of the form $f_{k}(e x)$, which are also solutions of (2). Therefore, $F_{k}\left(s_{1} x, s_{2} x, \ldots, s_{k} x\right)$ is a solution, and thus $F_{k} \in V$. Let

$$
\left(K^{*}\right)^{k}=\left\{\left(x_{1}, \ldots, x_{k}\right): x_{1}, \ldots, x_{k} \in K \backslash\{0\}\right\}
$$


Then $\left(K^{*}\right)^{k}$ is an Abelian group under multiplication by coordinates. It is easy to check that $V^{*}=\left\{\left.S\right|_{\left(K^{*}\right)^{k}}: S \in V\right\}$ is a translation invariant, closed, linear subspace of $C\left(\left(K^{*}\right)^{k}\right)$; i.e. it is a variety. Since $F_{k} \in V$, $V^{*}$ is nonzero.

Since the $b_{i}^{\prime} \mathrm{s}$ and $c_{i}^{\prime} \mathrm{s}$ are algebraic numbers, the field $K$ is a finite algebraic extension of the field $\mathbb{Q}$. Let $\beta_{1}, \ldots, \beta_{N}$ be a basis of $K$ as a linear space over $\mathbb{Q}$. If $E_{k}:\left(K^{*}\right)^{k} \rightarrow \mathbb{C}$ is $k$-additive, then

$$
\begin{aligned}
E_{k}(x, \ldots, x) & =E_{k}\left(r_{1} \beta_{1}+\ldots+r_{N} \beta_{N}, \ldots, r_{1} \beta_{1}+\ldots+r_{N} \beta_{N}\right)= \\
& =\left(r_{1}\right)^{k} E_{k}\left(\beta_{1}, \ldots, \beta_{1}\right)+\ldots+\left(r_{N}\right)^{k} E_{k}\left(\beta_{N}, \ldots, \beta_{N}\right)= \\
& =\sum_{\left(i_{1}, \ldots, i_{k}\right) \in I^{k}}\left(\prod_{s=1}^{k} r_{i_{s}}\right) E_{k}\left(\beta_{i_{1}}, \ldots, \beta_{i_{k}}\right)
\end{aligned}
$$

for every $r_{1}, \ldots, r_{N} \in \mathbb{Q}$ and $I=\{1, \ldots, N\}$.

Therefore, the values of $E_{k}$ at any point of $\left(K^{*}\right)^{k}$ are determined by the values $E_{k}\left(\beta_{1}, \ldots, \beta_{1}\right), \ldots, E_{k}\left(\beta_{N}, \ldots, \beta_{N}\right)$. Since the set of all functions mapping $\left\{\left(\beta_{1}, \ldots, \beta_{1}\right), \ldots,\left(\beta_{N}, \ldots, \beta_{N}\right)\right\}$ into $\mathbb{C}$ has dimension $N^{k}$, it follows that the set $\mathcal{E}_{k}$ of all $k$-additive functions defined on $\left(K^{*}\right)^{k}$ forms a finite dimensional vector space over $\mathbb{C}$. This implies that $V^{*}$, as a linear subspace of $\mathcal{E}_{k}$ is also finite dimensional.

Consequently, $V^{*}$ is a finite dimensional translation invariant, closed linear space of functions defined on $\left(K^{*}\right)^{k}$. By [8] and [2], it follows that every element of $V^{*}$ is a polynomial-exponential function.

In particular, $F_{k}=\sum_{j=1}^{L} P_{j} \cdot M_{j}$, where $P_{1}, \ldots, P_{L}$ are nonzero polynomials and $M_{1}, \ldots, M_{L}$ are distinct exponentials on $\left(K^{*}\right)^{k}$ with respect to the multiplication.

We shall prove that every exponential element of $V^{*}$ is of the form $\left.\phi_{1}\left(x_{1}\right) \phi_{2}\left(x_{2}\right) \ldots \phi_{k}\left(x_{k}\right)\right|_{\left(K^{*}\right)^{k}}$ where $\phi_{j}$ is an injective homomorphism for every $j \in\{1, \ldots, k\}$. We shall also prove that every exponential monomial element of $V^{*}$ is a constant multiple of an exponential. This will imply

$$
F_{k}\left(x_{1}, \ldots, x_{k}\right)=\sum_{j=1}^{L} c_{j} \cdot \phi_{1}\left(x_{1}\right) \ldots \phi_{k}\left(x_{k}\right)
$$

for every $x_{1}, \ldots, x_{k} \in V^{*}$. Putting $x_{1}=\ldots=x_{k}=x$ we obtain

$$
f_{k}(x)=F_{k}(x, \ldots, x)=\sum_{j=1}^{L} c_{j} \cdot \phi_{1}(x) \ldots \phi_{k}(x)
$$


for every $x \in K^{*}$. Since (9) is also true for $x=0$, this will complete the proof.

Let $M\left(x_{1}, x_{2}, \ldots, x_{k}\right)$ be an exponential element of $V^{*}$. Then $M$ is multiplicative in each coordinate, that is

$$
M\left(x_{1} y_{1}, x_{2} y_{2}, \ldots, x_{k} y_{k}\right)=M\left(x_{1}, x_{2}, \ldots, x_{k}\right) M\left(y_{1}, y_{2}, \ldots, y_{k}\right)
$$

for every $x_{i}, y_{i} \in K^{*}$. Therefore,

$$
\begin{aligned}
M\left(x_{1}, x_{2}, \ldots, x_{k}\right) & =M\left(x_{1}, 1, \ldots, 1\right) M\left(1, x_{2}, \ldots, 1\right) \cdots M\left(1,1, \ldots, x_{k}\right)= \\
& =m_{1}\left(x_{1}\right) m_{2}\left(x_{2}\right) \cdots m_{k}\left(x_{k}\right)
\end{aligned}
$$

for every $x_{1}, \ldots, x_{k} \in K^{*}$. We extend $m_{j}$ to $K$ by putting $m_{j}(0)=0$. Then each $m_{j}$ is an injective homomorphism, because it is additive by the $k$-additivity of $M$, and it is multiplicative as well.

Now we prove that each $P_{j}$ is constant.

Suppose, e.g. that $P_{1}$ is not constant. Then, by Lemma 2.1, there is a nonzero additive function $A$ on $\left(K^{*}\right)^{k}$ such that

$$
d\left(x_{1}, \ldots, x_{k}\right)=A\left(x_{1}, \ldots, x_{k}\right) \cdot m_{1}\left(x_{1}\right) \cdots m_{k}\left(x_{k}\right) \in V^{*} .
$$

The additivity of $A$ means that is,

$$
A\left(x_{1} \cdot y_{1}, x_{2} \cdot y_{2}, \ldots, x_{k} \cdot y_{k}\right)=A\left(x_{1}, x_{2}, \ldots, x_{k}\right)+A\left(y_{1}, y_{2}, \ldots, y_{k}\right)
$$

for every $x_{1}, \ldots, x_{n}, y_{1}, \ldots, y_{n} \in K^{*}$. We prove that in this case $d \equiv 0$ which is a contradiction.

Then $d\left(x_{1}, 1, \ldots, 1\right)=A\left(x_{1}, 1, \ldots, 1\right) m_{1}\left(x_{1}\right)$ is a polynomial exponential of the case of Theorem 2.3, therefore $d\left(x_{1}, 1, \ldots, 1\right)=0$ and since $m_{1}\left(x_{1}\right) \neq 0, A\left(x_{1}, 1, \ldots, 1\right)=0$. This is true for every coordinate. Using (10), we obtain that

$$
A\left(x_{1}, x_{2}, \ldots, x_{k}\right)=A\left(x_{1}, 1, \ldots, 1\right)+\cdots+A\left(1,1, \ldots, x_{k}\right)=0
$$

for every $x_{1}, x_{2}, \ldots, x_{k} \in K^{*}$, therefore $d$ must be zero.

Lemma 2.8. If $\phi_{1}, \ldots, \phi_{k}: K \rightarrow \mathbb{C}$ are injective homomorphisms then the product $\phi_{1} \cdots \phi_{k}$ is a solution of (2) if and only if

$$
\sum_{i=1}^{n} a_{i} \prod_{j \in J} \phi_{j}\left(b_{i}\right) \prod_{j^{\prime} \notin J} \phi_{j^{\prime}}\left(c_{i}\right)=0
$$

for every $J \subseteq\{1, \ldots, k\}$.

Proof. See [4, Lemma 3.4] 
Remark 2.9. In the special case when the functional equation can be written of the form (1) the situation is slightly simpler, since the condition (11) takes the following form:

$$
\sum_{i=1}^{n} a_{i} \phi_{j_{1}} \ldots \phi_{j_{s}}\left(b_{i}\right)=0
$$

for every choice of the integers $0 \leq j_{1}<\cdots<j_{s} \leq k(0 \leq s \leq k)$. Consequently, if we take the injective homomorphism solutions of (1), then forming the products at most $n-1$ of them gives a basis of the space of the solutions. Furthermore, the conditions (12) imply that every subproduct of a product which is a solution of (1) must be a solution, as well.

\section{TRIVIAL FUNCTIONAL EQUATIONS}

In this section we present another class of equations such that the set of its additive solutions is spanned by those solutions which are injective homomorphisms.

Theorem 3.1. The variety $V_{\mathbb{C}}$ generated by the automorphisms of $\mathbb{C}$ contains every additive function.

Proof. The variety $V_{\mathbb{C}}$ is the closure of the set of linear combinations of the automorphisms of $\mathbb{C}$. This means that whenever $f: \mathbb{C} \rightarrow \mathbb{C}$ is such that, for every $y_{1}, y_{2}, \ldots, y_{n} \in \mathbb{C}$ and $\varepsilon>0$ there are automorphisms $\phi_{1}, \phi_{2}, \ldots, \phi_{M}$ satisfying

$$
\left|f\left(y_{j}\right)-\sum_{m=1}^{M} c_{i} \cdot \phi_{m}\left(y_{j}\right)\right|<\varepsilon
$$

for every $j=\{1, \ldots, n\}$, then $f \in V_{\mathbb{C}}$. We have to show that every additive function on $\mathbb{C}$ has this property.

It is enough to prove that if $K$ is finitely generated, then the variety $V_{K}$, generated by the injective homomorphisms of $K$ into $\mathbb{C}$ contains every additive function on $K$. Indeed, for given points $y_{1}, y_{2}, \ldots, y_{n} \in$ $\mathbb{C}$, we may take the subfield $K$ generated by these points. If we can guarantee that the restriction of a given additive function $f$ to $K$ is in the variety $V_{K}$ for every finitely generated subfield $K$ of $\mathbb{C}$, then, by Proposition $2.2, V_{\mathbb{C}}$ contains the function $f$.

We will prove the following stronger statement by induction on the number of generators of $K$ : if a function $f \in V_{K}$ and the points 
$y_{1}, y_{2}, \ldots, y_{n} \in K$ are given, then there are injective homomorphisms $\phi_{1}, \phi_{2}, \ldots, \phi_{M}$ and there are complex numbers $c_{1}, \ldots, c_{M}$ such that

$$
f\left(y_{j}\right)=\sum_{m=1}^{M} c_{m} \cdot \phi_{m}\left(y_{j}\right)
$$

for every $j=\{1, \ldots, n\}$. It is clear that the statement is true for $K=\mathbb{Q}$, since every additive function on $\mathbb{Q}$ is of the form $c \cdot \operatorname{Id}$, where Id denotes the identity function of $\mathbb{Q}$. Let us assume that the statement is true for a finitely generated $K$. We prove the statement for $K(\beta)$ and for $K(t)$, where $\beta$ is an algebraic number, and $t$ is a transcendental number.

Case 1: $\beta$ is an algebraic number, and $|K(\beta): K|=n$. Then

$$
K(\beta)=\left\{a_{0}+a_{1} \beta+\cdots+a_{n-1} \beta^{n-1}: a_{i} \in K(i=0, \ldots, n-1)\right\} .
$$

Let the additive function $f: K(\beta) \rightarrow \mathbb{C}$ and the numbers $y_{1}, \ldots, y_{N} \in$ $K(\beta)$ be given.

Since every $y_{j}$ is a sum of terms of the form $b \cdot \beta^{i}(b \in K)$, therefore, by additivity, it is enough to represent $f$ at the points $b_{i, j} \cdot \beta^{i}$ for every $b_{i, j} \in K(j=1, \ldots, N, i=0, \ldots, n-1)$. For every fix $i$, the function $f\left(x \cdot \beta^{i}\right)$ is an additive function on $K$. Thus, by the induction hypothesis, there are injective homomorphisms $\phi_{1}, \ldots, \phi_{M}$ of $K$ into $\mathbb{C}$, and there are complex numbers $c_{i, m}$ such that

$$
f\left(b_{i, j} \cdot \beta^{i}\right)=\sum_{m=1}^{M} c_{i, m} \cdot \phi_{m}\left(b_{i, j}\right)
$$

for every $i=0, \ldots, n-1$ and $j=1, \ldots, N$. We need to represent every term on the right hand side of (16) by some linear combination of values of injective homomorphisms of $K(\beta)$ at the point $b_{i, j} \cdot \beta^{i}$. Let the roots of the minimal polynomial of $\beta$ be $\beta=\beta_{0}, \ldots, \beta_{n-1}$. We put $\psi_{m, k}\left(a_{0}+a_{1} \beta+\cdots+a_{n-1} \beta^{n-1}\right)=\phi_{m}\left(a_{0}\right)+\phi_{m}\left(a_{1}\right) \beta_{k}+\cdots+\phi_{m}\left(a_{n-1}\right) \beta_{k}^{n-1}$ for every $a_{0}, \ldots, a_{n-1} \in K$ and $k=0, \ldots, n-1$. Then $\psi_{m, k}$ is an injective homomorphism of $K(\beta)$ into $\mathbb{C}$ for every $m$ and $k$. We show that there are numbers $x_{m, k}$ such that

$$
f\left(b_{i, j} \beta^{i}\right)=\sum_{m=1}^{M} \sum_{k=0}^{n-1} x_{m, k} \psi_{m, k}\left(b_{i, j} \beta^{i}\right)
$$

that is,

$$
f\left(b_{i, j} \beta^{i}\right)=\sum_{m=1}^{M} \sum_{k=0}^{n-1} x_{m, k} \phi_{m}\left(b_{i, j}\right) \cdot \beta_{k}^{i}
$$


By (13), it is enough to find $x_{m, k}$ satisfying the equations

$$
c_{i, m} \cdot \phi_{m}\left(b_{i, j}\right)=\sum_{k=0}^{n-1} x_{m, k} \phi_{m}\left(b_{i, j}\right) \cdot \beta_{k}^{i}
$$

for every $i=0, \ldots, n-1, j=1, \ldots, N$ and $m=1, \ldots, M$. That is, $x_{m, k}$ has to satisfy

$$
c_{i, m}=\sum_{k=0}^{n-1} x_{m, k} \cdot \beta_{k}^{i}
$$

for every $i=0, \ldots, n-1$ and $m=1, \ldots, M$. Since the determinant of the system of equations (15) is nonzero (Vandermonde) for every fixed $m$, these systems are solvable. If $x_{m, k}$ is a solution, (14) shows that $f$ is represented on the set $\left\{b_{i, j} \beta^{i}\right\}$ as a linear combination of injective homomorphisms.

Case 2: If $t$ is a transcendental number, then every element of $K(t)$ is a rational function of the variable $t$ with coefficients from $K$.

Let $f: K(t) \rightarrow \mathbb{C}$ be an additive function. Let the rational functions $y_{1}, \ldots, y_{n} \in K(t)$ be given. Let $q(t)$ be a common multiple of the denominators of the rational functions $y_{i}$.

Since every $y_{j}$ is a sum of terms of the form $b \cdot t^{i} / q(t)(b \in K)$, therefore, by additivity, it is enough to represent $f$ at the points $b_{i, j}$. $t^{i} / q(t)$ for every $b_{i, j} \in K(j=1, \ldots, N, i=0, \ldots, n-1)$. For every fix $i$, the function $f\left(x \cdot t^{i} / q(t)\right)$ is an additive function on $K$. Thus, by the induction hypothesis, there are injective homomorphisms $\phi_{1}, \ldots, \phi_{M}$ of $K$ into $\mathbb{C}$, and there are complex numbers $c_{i, m}$ such that

$$
f\left(b_{i, j} \cdot t^{i} / q(t)\right)=\sum_{m=1}^{M} c_{i, m} \cdot \phi_{m}\left(b_{i, j}\right)
$$

for every $i=0, \ldots, n-1$ and $j=1, \ldots, N$. We need to represent every term on the right hand side of (13) by some linear combination of values of injective homomorphisms of $K(t)$ at the points $b_{i, j} \cdot t^{i} / q(t)$. Let $t_{0}, t_{2}, \ldots, t_{n-1}$ be algebraically independent elements over $K$, and put

$$
\psi_{m, k}(r(t))=\left(\phi_{m} \circ r\right)\left(t_{k}\right)
$$

for every $r \in K(t), m=1, \ldots, M$ and $k=0, \ldots, n-1$. Then $\psi_{m, k}$ is an injective homomorphism of $K(t)$ into $\mathbb{C}$ for every $m$ and $k$. We 
show that there are numbers $x_{m, k}$ such that

$$
f\left(b_{i, j} t^{i} / q(t)\right)=\sum_{m=1}^{M} \sum_{k=0}^{n-1} x_{m, k} \psi_{m, k}\left(b_{i, j} t^{i} / q(t)\right) ;
$$

that is,

$$
f\left(b_{i, j} t^{i} / q(t)\right)=\sum_{m=1}^{M} \sum_{k=0}^{n-1} x_{m, k} \phi_{m}\left(b_{i, j}\right) \cdot \frac{t_{k}^{i}}{\phi_{m}\left(q\left(t_{k}\right)\right)} .
$$

By (13), it is enough to find $x_{m, k}$ satisfying the equations

$$
c_{i, m} \cdot \phi_{m}\left(b_{i, j}\right)=\sum_{k=0}^{n-1} x_{m, k} \phi_{m}\left(b_{i, j}\right) \cdot \frac{t_{k}^{i}}{\phi_{m}\left(q\left(t_{k}\right)\right)}
$$

for every $i=0, \ldots, n-1, j=1, \ldots, N$ and $m=1, \ldots, M$. Let $z_{m, k}$ satisfy

$$
c_{i, m}=\sum_{k=0}^{n-1} z_{m, k} \cdot t_{k}^{i}
$$

for every $i=0, \ldots, n-1$ and $m=1, \ldots, M$. Since the determinant of the system of equations (19) is nonzero (Vandermonde) for every fixed $m$, these systems are solvable. If $z_{m, k}$ is a solution, then put $x_{m, k}=z_{m, k} \cdot \phi_{m}\left(q\left(t_{k}\right)\right)$. Then (17) shows that $f$ is represented on the set $\left\{b_{i, j} t^{i} / q(t)\right\}$ as a linear combination of injective homomorphisms.

This completes the proof.

\section{Further CASES}

Theorem 3.1 might suggest that if there are infinitely many injective homomorphisms which are solutions of (2), then the variety generated by these injective homomorphisms contains every additive solution as well. Now we show that this is not true (see Theorem 4.3 below).

Definition 4.1. Let $K$ denote a field over $\mathbb{Q}$. We say that a function $d: K \rightarrow \mathbb{C}$ is a derivation if it has the following two properties:

(1) $d(x+y)=d(x)+d(y)$ and

(2) $d(x y)=d(x) y+x d(y)$

for every $x, y \in K$.

Lemma 4.2. Let $K \subset L$ be subfields of $\mathbb{C}$. If $d$ is derivation on $K$, then it can be extended to $L$ as a derivation. 
Proof. Well-known. See, e.g. [7, Proposition, p. 154].

Theorem 4.3. If $K \subset \mathbb{C}$ is a field which contains a transcendental number, then there exists a linear functional equation

$$
\sum_{i=1}^{n} a_{i} f\left(b_{i} x+y\right)=0
$$

such that $b_{i} \in K$ for every $i=1, \ldots, n$, and there exists an additive solution which is not contained by the variety generated by the injective homomorphisms which are solutions.

Proof. Let us suppose that $t \in K$ is a transcendental number and $a_{1}, \ldots, a_{n}$ are nonzero complex numbers satisfying

$$
\sum_{i=1}^{n} a_{i}=0, \sum_{i=1}^{n} a_{i} t^{i}=0, \sum_{i=1}^{n} a_{i} i t^{i-1}=0 .
$$

We will prove that in this case the functional equation

$$
\sum_{i=1}^{n} a_{i} f\left(t^{i} x+y\right)=0
$$

satisfies the requirements.

Every element of $\mathbb{Q}(t)$ is a rational function with rational coefficients of the variable $t$. The operation $d_{0}=\frac{\partial}{\partial t}$ is a well-defined derivation on $\mathbb{Q}(t)$. It follows from Lemma 4.2 that we can extend $d_{0}$ from $\mathbb{Q}(t)$ to $K$ as a derivation. Then $d_{0}$ is a solution of (21). Indeed,

$$
\begin{aligned}
& \sum_{i=1}^{n} a_{i}\left(d_{0}\left(t^{i} x+y\right)\right)=\sum_{i=1}^{n} a_{i} d_{0}\left(t^{i}\right) x+\sum_{i=1}^{n} a_{i} t^{i} d_{0}(x)+\sum_{i=1}^{n} a_{i} d_{0}(y)= \\
& =\sum_{i=1}^{n} a_{i} i t^{i-1} d_{0}(t) x+\sum_{i=1}^{n} a_{i} t^{i} d_{0}(x)+\sum_{i=1}^{n} a_{i} d_{0}(y)=0
\end{aligned}
$$

by $(20)$.

Let $V$ denote the variety generated by those injective homomorphisms which are solutions of (21) on $K$.

Suppose $\phi \in V$ is an injective homomorphism. Then, by (20), we have $\sum_{i=1}^{n} a_{i} \phi(t)^{i}=0$, and thus $\phi(t)$ is a root of the polynomial $p(x)=$ $\sum_{i=1}^{n} a_{i} x^{i}$. Let $u_{1}, \ldots, u_{k}$ be the distinct roots of $p$. Suppose $\phi(t)=u_{i}$. If $\psi \in V$ is another injective homomorphism with $\psi(t)=u_{i}$, then restrictions of $\phi$ and $\psi$ to $\mathbb{Q}(t)$ coincide. This easily implies that the family of restrictions $W=\left\{\left.g\right|_{\mathbb{Q}(t)}: g \in V\right\}$ is finite dimensional. It 
is clear that if $\phi_{1}, \ldots, \phi_{k}$ are fixed injective homomorphisms such that $\phi_{j}(t)=u_{j}(j=1, \ldots, k)$, then the restrictions $\left.\phi_{j}\right|_{\mathbb{Q}(t)}$ form a basis of $W$ as a linear space.

Suppose $d_{0} \in V$. Then there are complex numbers $c_{1}, \ldots, c_{k}$ such that

$$
\sum_{j=1}^{k} c_{j} \phi_{j}(w)=d_{0}(w)
$$

for every $w \in \mathbb{Q}(t)$. Applying (23) with $w=t^{i}$ and using $d_{0}\left(t^{i}\right)=i \cdot t^{i-1}$, we find

$$
\sum_{j=1}^{k} c_{j} \phi_{j}\left(t^{i}\right)=\sum_{j=1}^{k} c_{j} u_{j}^{i}=i \cdot t^{i-1}
$$

for every $i=1,2, \ldots$ Multiplying by $z^{i}$ and summing for $i=1,2, \ldots$. we obtain

$$
\sum_{j=1}^{k} c_{j} \sum_{i=1}^{\infty} u_{j}^{i} z^{i}=\sum_{i=1}^{\infty} i \cdot t^{i-1} z^{i},
$$

as an equation between formal power series. However, for $z$ small enough both power series are convergent, and we obtain

$$
\sum_{j=1}^{k} c_{j} \frac{u_{j} z}{1-u_{j} z}=\frac{\partial}{\partial t} \cdot \frac{1}{1-t z}=-\frac{z}{(1-t z)^{2}}
$$

for every $|z|<\delta$. The functions was defined with power series and the intersection of domains of convergence contain a set that has a non-isolated point. These implies that the functions are equal in the union of the domains using Uniqueness Principle. This implies is that equation (24) is an identity on the whole complex plane, since the last term of equation (24) is a meromorphic function on the whole space with a pole singularity in $z=1 / t$. Therefore,

$$
\sum_{j=1}^{k} c_{j} \frac{(1-t z)^{2} u_{j} z}{1-u_{j} z}=-z
$$

holds for every $z \neq 1 / t$ and if $z \rightarrow \frac{1}{t}$ the left side tends to 0 , but the right is not.

This contradiction shows that $d_{0} \notin V$, which completes the proof.

Example It is easy to check that the function equation

$$
f\left(t^{2} x+y\right)-2 t f(t x+y)+t^{2} f(x+y)-(t+1)^{2} f(y)=0
$$

satisfies the conditions of Theorem 4.3. 


\section{REFERENCES}

[1] J. A. Baker, A general functional equation and its stability, Proc. Am. Math. Soc. 133 (2004), no. 6, 1657-1664

[2] M. Engert, Finite dimensional translation invariant subspaces, Pacific J. Math., 32 (1970), 333-343.

[3] M. Kuczma, An introduction to the Theory of Functional Equations and Inequalities, (1985).

[4] G. Kiss and A. Varga, Existence of nontrivial solutions of linear functional equations, to appear in Aequationes Math.

[5] M. Laczkovich, Polynomial mappings on Abelian groups, Aequationes Math. 68 (2004), 177-199

[6] S. Mazur and W. Orlicz, Grundlegende Eigenschaften der polynomischen Operationen, Fund. Math. (1939), 42-50.

[7] M. Rosenlicht, Liouville's theorem on functions with elementary integrals, Pacific J. Math. 24 No. 1 (1968), 153-161.

[8] L. Székelyhidi, Note on exponential polynomials, Pacific J. Math. 103 (1982), 583-587.

[9] L. Székelyhidi, On a class of linear functional equations, Publ. Math. (Debrecen) 29 (1982), 19-28.

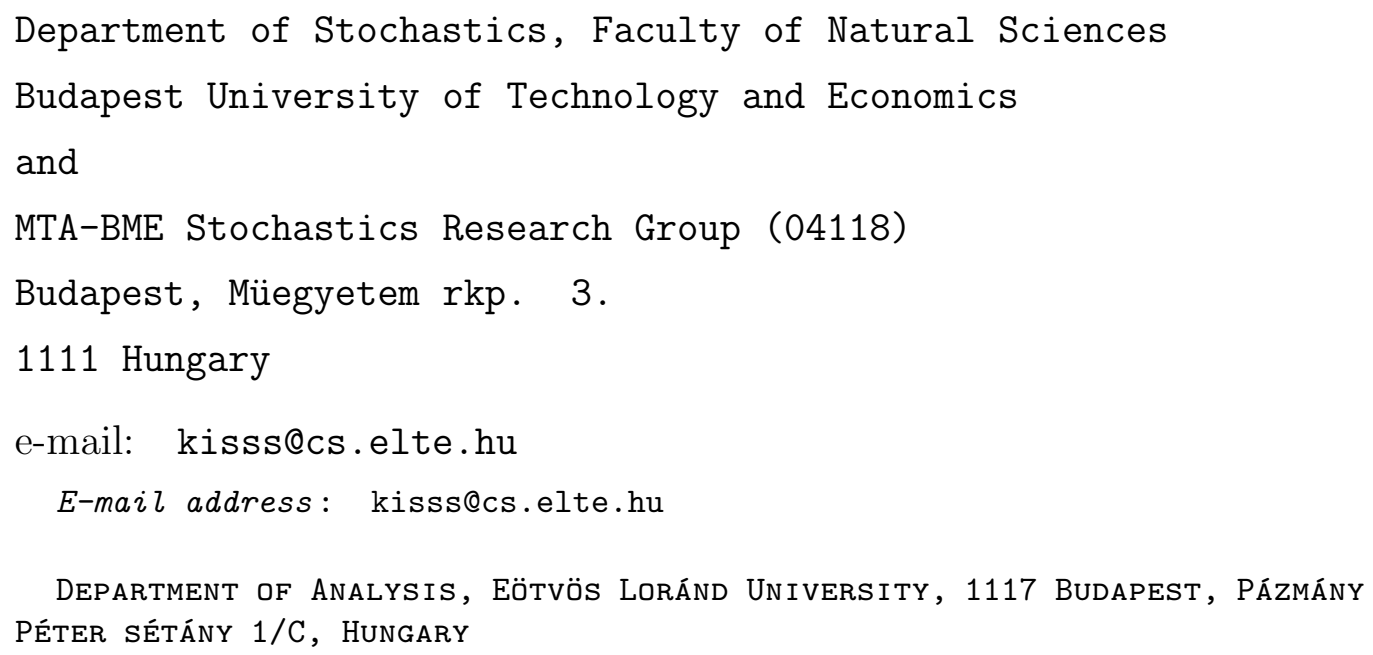

